\title{
Criminologie
}

\section{La contrainte des choix : un regard rétrospectif}

\section{Margaret Shaw et Kelly Hannah-Moffat}

Volume 35, numéro 2, automne 2002

Femmes et enfermement au Canada : une décennie de réformes

URI : https://id.erudit.org/iderudit/008290ar

DOI : https://doi.org/10.7202/008290ar

Aller au sommaire du numéro

Éditeur(s)

Les Presses de l'Université de Montréal

ISSN

0316-0041 (imprimé)

1492-1367 (numérique)

Découvrir la revue

Citer cet article

Shaw, M. \& Hannah-Moffat, K. (2002). La contrainte des choix : un regard rétrospectif. Criminologie, 35(2), 53-72. https://doi.org/10.7202/008290ar

\section{Résumé de l'article}

Cet article présente une réflexion sur les thèmes et les présupposés qui étaient à la base du Rapport du Groupe d'étude sur les femmes purgeant une peine fédérale (La création de choix, 1990). L'incompatibilité entre, d'une part, les idéaux féministes et autochtones et, d'autre part, les pratiques d'enfermement est l'une des difficultés inhérentes aux efforts de reconstruction des modèles pénaux dont nous traiterons. Plusieurs questions reliées au processus de mise en oeuvre des idées contenues dans le rapport ou aux conséquences imprévues de la réforme sont préoccupantes. Puisqu'il est impossible d'aborder ici toutes ces questions, nous traiterons des changements qui ont fait suite à La création de choix sous deux aspects plus spécifiques. Dans un premier temps, la situation des femmes perçues comme violentes ou "difficiles à gérer " sera examinée. Nous traiterons ensuite du cas des détenues classées à un niveau de sécurité minimal et de la stratégie communautaire. Nous nous interrogeons, dans cet article, sur ce que signifie réellement, aujourd'hui, l'idée d'offrir des " choix " à ces femmes détenues; nous cherchons à voir jusqu'à quel point la possibilité de choisir leur est vraiment offerte.
Ce document est protégé par la loi sur le droit d'auteur. L'utilisation des services d’Érudit (y compris la reproduction) est assujettie à sa politique d'utilisation que vous pouvez consulter en ligne.

https://apropos.erudit.org/fr/usagers/politique-dutilisation/ 


\title{
La contrainte des choix : un regard rétrospectif ${ }^{1}$
}

\author{
Margaret Shaw \\ Directrice - analyse et échange \\ Centre international pour la prévention de la criminalité \\ Montréal \\ shaw@crime-prevention-intl.org \\ Kelly Hannah-Moffat \\ Professeure \\ Département de Sociologie \\ Université de Toronto à Missisauga \\ khmoffat@utm.utoronto.ca
}

RÉSUMÉ - Cet article présente une réflexion sur les thèmes et les présupposés qui étaient à la base du Rapport du Groupe d'étude sur les femmes purgeant une peine fédérale ( $L a$ création de choix, 1990). L'incompatibilité entre, d'une part, les idéaux féministes et autochtones et, d'autre part, les pratiques d'enfermement est l'une des difficultés inhérentes aux efforts de reconstruction des modèles pénaux dont nous traiterons. Plusieurs questions reliées au processus de mise en œuvre des idées contenues dans le rapport ou aux conséquences imprévues de la réforme sont préoccupantes. Puisqu'il est impossible d'aborder ici toutes ces questions, nous traiterons des changements qui ont fait suite à La création de choix sous deux aspects plus spécifiques. Dans un premier temps, la situation des femmes perçues comme violentes ou «difficiles à gérer» sera examinée. Nous traiterons ensuite du cas des détenues classées à un niveau de sécurité minimal et de la stratégie communautaire. Nous nous interrogeons, dans cet article, sur ce que signifie réellement, aujourd'hui, l'idée d'offrir des «choix» à ces femmes détenues; nous cherchons à voir jusqu'à quel point la possibilité de choisir leur est vraiment offerte.

ABSTRACT - This paper reflects on and re-evaluates some of the themes and assumptions informing the 1990 Report of the Task Force on Federally Sentenced Women - Creating Choices. We discuss some of the intrinsic difficulties associated with attempts to reconstruct penal regimes for women including the incompatibility of feminist and Aboriginal ideals with institutional practices of imprisonment. Since it is not possible to cover all

1. Texte traduit par Julie Cantin. 
of the concerns about the implementation process and the largely unintended consequences of this reform, we focus on the impact of these changes for women who are seen as violent or "difficult to handle», and the fate of minimum security women and the community strategy. The article considers what giving those women prisoners «choices» has come to signify, and how far they have been offered choice.

\section{Introduction}

Le Groupe d'étude sur les femmes purgeant une peine fédérale avait comme leitmotiv la question des choix, comme l'indique le titre de son rapport, La création de choix (1990). Celui-ci était porteur d'un nouveau message d'espoir et reconnaissait la spécificité des prisons pour femmes. Il promettait en outre un avenir vraiment différent. Le rapport et son groupe d'étude se distinguaient clairement des autres commissions et rapports portant sur les «populations spéciales» mandatés par le Service correctionnel du Canada (SCC) à cette époque. La composition du groupe d'étude, le contenu du rapport ainsi que ses éventuelles conséquences illustraient une réelle volonté d'innovation. Le groupe était en effet constitué d'une proportion importante de femmes et de représentants issus de milieux non gouvernementaux, il a eu recours à des types novateurs de consultations et de recherches et avait des visées très ambitieuses quant à ses recommandations et à l'étendue de leur application.

Le rapport La création de choix faisait état d'une nouvelle philosophie de l'enfermement des femmes au Canada. Il a de plus exposé les principes de la construction et de la gestion de cinq nouvelles prisons destinées uniquement aux femmes. Le Groupe d'étude y proposait en conséquence la fermeture de la prison des femmes de Kingston, en Ontario - le seul pénitencier pour femmes au Canada - et ce, au plus tard pour l'année 1995. Un modèle correctionnel centré sur les femmes et sensible aux différences culturelles a été élaboré en vue de remplacer l'ancien modèle. Le Groupe d'étude a d'ailleurs tenu compte de plusieurs questions qui préoccupaient, depuis longtemps, des détenues, des femmes autochtones, des réformatrices féministes, certains avocats et fonctionnaires. Le rapport constituait un exemple de peines innovatrices et progressistes. Grâce à lui, le Canada s'est retrouvé dans une position unique. Il devenait l'un des premiers pays à tenter de développer, sur la base de principes féministes, un modèle de prisons pour les femmes. C'était également l'un des premiers à reconnaitre l'incroyable fardeau que fait porter le système correctionnel aux femmes autochtones et la nécessité d'agir à propos de leur surreprésentation. Le rapport a soulevé beaucoup d'intérêt dans 
d'autres pays et a influencé, pendant un certain temps, certains gouvernements provinciaux, lesquels ont alors repensé leurs politiques concernant les prisons pour femmes (la Nouvelle-Écosse et l'Ontario ont produit des rapports en 1991 et en 1995 (Shaw, 1996)).

L'anniversaire du rapport La création de choix constitue un moment privilégié pour réfléchir sur les choix qu'il proposait, sur la signification réelle de l'idée d'offrir des choix aux détenues et sur l'étendue véritable de cette possibilité de choisir. Nous utilisons le rapport en tant que base d'analyse des difficultés associées aux tentatives de reconstruction des modèles pénaux s'adressant aux femmes. Onze ans après La création de choix, il est possible de prendre connaissance de l'incompatibilité entre, d'une part, les idéaux féministes et autochtones et, d'autre part, les pratiques institutionnelles d'incarcération, de faire ressortir les décisions opérationnelles ayant contribué à l'érosion des idéaux de La création de choix, puis de retracer les sources de pression et les événements particuliers qui ont eu pour effet de limiter davantage les changements prévus par le rapport. Les choses sont toujours plus claires rétrospectivement.

Le rapport a influencé plusieurs aspects du système correctionnel. Il est impossible de traiter chacun d'eux dans le cadre de cet article. Deux cas particuliers seront donc examinés, soit la situation des femmes perçues comme violentes ou «difficiles à gérer» et celle des détenues à sécurité minimale et de la stratégie communautaire.

\section{Le Groupe d'étude et La création de choix}

Les enquêtes sur les femmes purgeant une sentence d'emprisonnement fédérale et les vains efforts pour réformer le traitement pénal des femmes ont une longue histoire au Canada. Cette dernière a pris fin en 1989 avec la nomination du Groupe d'étude sur les femmes purgeant une peine fédérale (Berzins et Collette-Carrière, 1979; Berzins et Hayes, 1987; Cooper, 1987; Shaw, 1991; Biron, 1992; Hannah-Moffat 1997; 2001). Les caractéristiques du Groupe d'étude étaient inhabituelles, ce dernier étant composé de membres du secteur communautaire et du gouvernement, de groupes autochtones et de minorités, et même de détenues. Plusieurs membres du Groupe avaient recours à une perspective féministe en prenant comme point de départ l'expérience des femmes. Ils affirmaient que les besoins des femmes étaient différents de ceux des hommes et que les approches correctionnelles traditionnelle, basées sur la population masculine, étaient inappropriées. 
Le Groupe d'étude a basé une partie importante de son analyse sur les différences entre hommes et femmes dans le système pénal. Les femmes détenues (comme les hommes) sont en majorité jeunes, pauvres, souvent sans emploi et disposent de peu de ressources et de faibles revenus; elles sont peu scolarisées et ont rarement des compétences de travail reconnues; les infractions qu'elles ont commises comprennent par ailleurs généralement la consommation d'alcool et de drogue (Pollock-Byrne, 1990; Shaw, 1994b; Statistique Canada, 1998; 1999). D'autre part, elles ont des besoins très différents de ceux des détenus masculins : deux tiers d'entre elles ont des enfants à charge, souvent dans une situation de monoparentalité; leurs besoins en termes de soins de santé sont différents et souvent plus grands que ceux des hommes; leur passé criminel est généralement moins étendu et moins sérieux que celui des détenus de genre masculin; elles risquent moins d'être à nouveau trouvées coupables d'un crime et sont vues comme un «meilleur risque»; elles ont par ailleurs été victimes d'abus de façon beaucoup plus importante, qu'il s'agisse d'abus physique, sexuel ou émotionnel, à l'enfance et à l'âge adulte. Les femmes autochtones sont encore plus surreprésentées que les détenus masculins de la même origine culturelle — de 20 à $25 \%$ des femmes en prison sont autochtones ${ }^{2}$. Globalement, les détenues sont, comparativement aux détenus, moins éduquées et moins formées en vue d'une profession; elles sont plus pauvres et dépendantes de la sécurité du revenu; elles ont plus de chances d'être sans emploi lors de leur admission en prison et d'être diagnostiquées comme ayant des problèmes de santé mentale (Hamelin, 1989; Shaw, 1994a et b).

En plus des différences «factuelles», le Groupe d'étude a reconnu que les femmes vivaient et réagissaient à la prison différemment des hommes. Elles ont tendance à ressentir les «douleurs de l'emprisonnement» de façon plus prononcée et à exprimer leurs sentiments de façon différente (Liebling, 1994). Pour les femmes, les relations avec les enfants, la famille et les amis sont centrales et leurs relations en prison sont plus intimes que celles des hommes. En général, il y a moins de violence manifeste dans la prison, mais plus de chances que les femmes se blessent, se mutilent ou tentent de se suicider (Shaw, 1994a et b). Le contrôle des détenues par médication n'est pas nouveau en prison (de même qu'à extérieur), et on note

2. Un sondage de 1996 sur les populations des prisons à travers le Canada a révélé que $20 \%$ des détenues fédérales étaient autochtones; cette proportion est de $23 \%$ dans les prisons provinciales et territoriales. Pour les hommes, la proportion d'autochtones était de $14 \%$ et de $18 \%$ (Statistique Canada, 1999). 
un recours plus élevé aux procédures disciplinaires pour des infractions mineures auxquelles on ne donnerait pas suite dans les établissements pour hommes (Mandaraka-Sheppard, 1986; Sim, 1990). Les femmes font donc souvent l'objet de mesures disciplinaires pour des comportements auxquels on ne réagirait pas dans une prison masculine. Elles sont d'ailleurs fréquemment perçues comme étant plus difficiles à gérer que les hommes, plus «verbales» et émotionnelles. Le traitement et le contrôle différentiels des détenues sont par ailleurs associés aux standards normatifs de la classe moyenne et aux attentes sociales selon le genre (Hamelin, 1989; Bertrand et al., 1998; Hannah-Moffat, 2001).

Bref, les types d'infractions pour lesquelles les femmes sont condamnées, le contexte de la commission de l'infraction, leurs expériences à l'extérieur et à l'intérieur de la prison, de même que la façon dont elles réagissent à l'emprisonnement sont à la fois quantitativement et qualitativement différents de ceux des hommes (Hannah-Moffat et Shaw, 2001b). Il y a aussi des différences entre femmes en termes d'expériences concernant le racisme et la pauvreté. Pour le Groupe d'étude, ces différences ont fait ressortir l'importance de considérer l'emprisonnement des femmes de façon indépendante de celui des hommes, ce qui n'empêche pas que les établissements pour hommes puissent bénéficier des changements apportés au traitement des femmes.

Le Groupe d'étude a donc défendu une approche centrée sur les femmes pour guider la mise en place de nouveaux établissements, modèles et programmes. Ses membres ont recommandé la fermeture de la prison des femmes de Kingston, la construction de cinq nouveaux établissements régionaux et d'un pavillon de ressourcement (ou loge de guérison) pour les femmes autochtones, ainsi que le développement parallèle d'une stratégie de libération communautaire. Le rapport a élaboré cinq principes centrés sur les femmes dont les nouveaux établissements et les nouvelles politiques s'inspireraient ${ }^{3}$ :

i) L'autonomisation par des programmes visant à accroître l'estime personnelle des femmes et à leur donner un plus grand contrôle sur leur vie.

ii) La mise sur pied de programmes et d'installations offrant des choix significatifs et des opportunités réalistes pour que les femmes puissent développer leur potentiel. 
iii) Le principe selon lequel il faudrait traiter les femmes avec respect et dignité, en incluant le respect de leur culture et de leur religion.

iv) Le développement d'un environnement de soutien, avec accès à une nourriture saine, de l'air frais, de la lumière, de l'espace, de l'intimité et des relations de soutien, constituait un principe essentiel.

v) La communauté, les organisations bénévoles et les femmes ellesmêmes devraient avoir une responsabilité partagée pour prendre soin d'elles et de leur développement. Ce ne serait plus la seule responsabilité du système correctionnel.

Le rapport esquissait les grandes lignes d'un projet de nouvelles prisons régionales composées de petites maisons de style résidentiel pouvant loger de six à dix femmes. On proposait que le nombre d'employés soit suffisamment élevé pour éviter de devoir se fier à des mesures de sécurité matérielles. Une «maison» aurait tout de même un niveau de sécurité plus élevé et serait destinée à un petit nombre de femmes qui y resteraient sur une base temporaire. Les installations ne nécessiteraient aucune clôture, sauf dans quelques cas afin de garder les gens à l'extérieur. Tous les employés seraient sélectionnés avec attention, en fonction notamment de leur sensibilité, et recevraient une formation obligatoire sur des aspects tels que le counselling, la communication, la négociation, les différences culturelles, le racisme et le sexisme.

Le rapport suggérait en outre la mise sur pied de programmes holistiques, c'est-à-dire des programmes qui devraient refléter la nature interreliée des besoins et expériences des femmes. Chaque établissement fournirait des programmes de fond construits spécialement pour les femmes détenues, plutôt qu'adaptés à partir des programmes pour hommes (counselling individuel ou de groupe pour la violence familiale, les victimes d'inceste et les habiletés quotidiennes de base), ainsi que des services de santé physique et mentale, des programmes concernant la toxicomanie, l'éducation, la formation professionnelle, des programmes récréatifs, spirituels et culturels. Le pavillon de ressourcement serait quant à lui développé et dirigé par des autochtones; les employés seraient également autochtones, liés à une communauté autochtone des environs et gouvernés par un Conseil des Aînés.

La deuxième partie, essentielle, du plan prévu par la commission spéciale porte sur le développement d'une série de ressources communautaires pour les femmes, ressources qui pourraient continuer à fournir les pro- 
grammes offerts à l'intérieur des établissements, tout en assurant une forme de support lors du retour dans la communauté.

Bref, la rhétorique et les intentions du rapport se distinguaient clairement des anciennes initiatives. On mettait fortement l'accent sur l'expérience des femmes en tant qu'anciennes victimes d'abus, alors que les questions de violence, de crises et de perturbations avaient volontairement été laissées de côté. Globalement, les détenues étaient présentées comme non violentes; on proposait, comme solution à d'éventuelles difficultés, un accroissement du nombre d'employés présents. Les femmes autochtones membres du Groupe d'étude ont par ailleurs eu un impact considérable en revendiquant la reconnaissance de leurs expériences de racisme, d'oppression et de violence (Sugar et Fox, 1989; mais voir Monture, 2000). Mais par-dessus tout, le rapport soulignait que chaque femme était unique par son histoire de vie, ses expériences et ses besoins, et que chacune devrait donc se voir offrir des choix en termes d'étendue de services et de programmes appropriés à ses besoins.

Le gouvernement a accepté les recommandations du Groupe d'étude et a accordé un budget de 50 millions de dollars pour sa mise en œuvre. Les nouvelles prisons à Truro, en Nouvelle-Écosse, à Kitchener, en Ontario, à Edmonton, en Alberta, à Joliette, au Québec et la Okimaw Ochi Healing Lodge (pavillon de ressourcement) à Maple Creek, en Saskatchewan, ont finalement ouvert leurs portes entre la fin de l'année 1995 et le printemps $1997^{4}$.

\section{Examen rétrospectif des problèmes d'implantation : un idéal inatteignable ou dangereux?}

À première vue, le Canada donne l'impression d'être à la fine pointe des orientations correctionnelles pour femmes avec son nouveau modèle centré sur elles, mais, malgré l'adoption de ce modèle, plusieurs problèmes administratifs et idéologiques subsistent. D'après ce bref sommaire du contenu du rapport, il est clair que La création de choix représentait une nouvelle voie, en rupture avec les anciennes pratiques pénales. La pensée

4. La Colombie-Britannique devait initialement être incluse, mais elle a renouvelé son entente d'échange de services avec le gouvernement fédéral en 1990. Une nouvelle prison pour femmes, conçue cependant selon le modèle traditionnel, a été construite à Burnaby pour recevoir des femmes sous juridiction provinciale et fédérale. Burnaby est demeuré en marge des développements concernant les détenues fédérales. 
des membres du Groupe d'étude reflétait avant tout le désir d'une restructuration fondamentale de l'enfermement des femmes au niveau fédéral et cela en tenant prioritairement compte des différences entre les genres et entre les cultures. On pourrait croire que le Groupe a atteint cet objectif du fait qu'il a contribué à la production d'un nouveau discours sur les sanctions pénales pour femmes; cependant, il reste à savoir jusqu'à quel point les idéaux de La création de choix ont ou n'ont pas changé les pratiques pénales. La rhétorique dominante du SCC soutient qu'une pratique pénale alternative, sensible aux différences culturelles, est maintenant en opération. On note cependant l'émergence d'un courant de littérature faisant de plus en plus ressortir les décisions prises lors du processus d'implantation du rapport, l'impact de certains événements sensationnels, les différentes interprétations des recommandations du rapport, les incohérences théoriques de ce dernier et ses conséquences imprévues (Biron, 1992; Faith, 1995 ; 1999; Hannah-Moffat, 1995; 2001 ; à paraître; Shaw, 1996; Hannah-Moffat et Shaw, 2000a; 2001 ; Hayman, 2000; à paraître; Kendall, 2000; Monture, 2000).

\section{Idéalisme déplacé, conséquences imprévues et barrières organisationnelles}

À l'intérieur comme à l'extérieur du service correctionnel, on a affirmé que le rapport La création de choix, même s'il constituait une vision innovatrice du changement, était peu réaliste, inatteignable ou incompatible avec les modèles pénaux. Certains ont souligné les contradictions entre la philosophie et les pratiques actuelles (Hannah-Moffat, 2001). Le SCC maintient que la plupart des femmes vont bénéficier du modèle des nouveaux établissements régionaux, mais il reconnaît également se trouver dans une phase d'apprentissage à laquelle sont associés plusieurs inconvénients, comme c'est toujours le cas lors de l'implantation d'un nouveau modèle. Plus récemment, le SCC a affirmé que plusieurs des idéaux de La création de choix n'étaient pas faciles à opérationnaliser et étaient, dans certains cas, trop ambitieux (Reynolds, 1996; Drouin, 1997; Stableforth, 1997). Plusieurs soutiennent maintenant (et ce, à la fois à l'intérieur et à l'extérieur du secteur correctionnel) que la prison centrée sur les femmes présentée dans le rapport constitue un idéal inatteignable. À l'intérieur du SCC, le rapport La création de choix est maintenant perçu et présenté comme une simple intention philosophique. Même si le modèle correctionnel contenu dans le rapport était et reste une alternative attrayante, 
innovatrice et progressiste par rapport aux régimes carcéraux traditionnels, la punitive et sinistre réalité disciplinaire des sanctions persiste (Hannah-Moffat et Shaw 2000b). Des expériences récentes ont illustré le degré de difficulté de l'opérationnalisation de ces idéaux.

Dans une autre perspective, on pourrait affirmer que les idéaux de $L a$ création de choix étaient atteignables, mais que le rapport a été «compromis» au cours de sa mise en œuvre par des événements exceptionnels qui n'ont pas été anticipés, par des pressions externes et par des contraintes organisationnelles. L'exclusion complète, au cours du processus d'implantation, de plusieurs membres en règle du Groupe d'étude passe souvent inaperçue. Plusieurs partenaires importants ou groupes d'intérêts n'ont pas été consultés à propos de décisions opérationnelles cruciales, telles que la sélection des sites. Des modifications ont été apportées au plan initial. On a ainsi ajouté des cellules sécuritaires, construit des clôtures et, dans au moins une des prisons, éliminé des éléments clés tels que la garderie ou le gymnase. Ces décisions ont été prises par le gouvernement sur la base de diverses raisons telles que la réduction des coûts, les pressions exercées par des citoyens locaux, l'emploi et la création d'emplois (Hannah-Moffat, 1995). Les pratiques d'exclusion ont quant à elles entrainé le retrait d'un participant clé du Groupe d'étude - l'Association canadienne des sociétés Elizabeth Fry (ACSEF) — de la phase d'implantation (Shaw, 1993).

Au moment où la mise en oeuvre du rapport était en cours, des événements internes et externes au SCC ont affecté la vision initiale. On a assisté à la naissance d'un sentiment de colère de la part du public à propos de certains sites prévus pour les nouvelles prisons. Au cours du processus de sélection des sites, d'importantes campagnes visant à prévenir la construction de prisons ont été organisées. La colère a été amplifiée par le procès Bernardo/Homolka, qui mettait l'accent sur le potentiel de violence des femmes, aspect que La création de choix s'était efforcé d'éviter par sa présentation des femmes comme étant d'abord des victimes de violence. Un sentiment de frustration concernant la légitimité des conditions de vie des détenues a également fait surface dans le discours public. Cela a contribué à un ressentiment croissant, lequel a été bien articulé dans les médias écrits. De plus en plus, ceux-ci évoquaient les principes de moindre éligibilité en affirmant que les prisonniers devraient être punis plutôt que de bénéficier de programmes d'aide et de conditions matérielles auxquels d'honnêtes citoyens n'ont même pas accès.

Les événements survenus en avril 1994 à la prison des femmes de Kingston semblent confirmer le changement d'attitudes et de perceptions 
dans le discours sur les femmes détenues. Un conflit entre six détenues et un certain nombre de gardiens a entrâné une suite d'actions qui ne furent pas sans conséquences. Ces moments difficiles ont donné lieu à la ségrégation de détenues, à des perturbations constantes, au déni des droits et privilèges des détenues, à l'événement très publicisé où des femmes ont été brutalisées et fouillées par une équipe d'intervention d'urgence masculine, au transfert de celles-ci et à leur mise en isolement (pour une période pouvant aller jusqu'à huit mois dans certains cas), aux éventuelles auditions disciplinaires, bref à davantage de punition (Faith, 1995; Arbour, 1996; Frigon, 1999; Shaw, 1999). Les changements d'attitudes ont été aggravés par une série d'événements disparates dans deux des nouvelles prisons qui avaient ouvert leurs portes en 1996, soit des cas de mutilation, d'évasion, un suicide (qui était peut-être un meurtre) et la destruction de cellules. Ces événements ont rendu caduque l'idée selon laquelle les nouveaux modèles et institutions élimineraient en eux-mêmes la plupart des difficultés qui survenaient dans l'ancien pénitencier.

La réaction du public à ces divers événements fut un accroissement du sentiment de colère et de l'effet de scandale; à cela s'ajoutait une demande prévisible de mesures de sécurité additionnelles dans les nouvelles institutions. Les nouvelles installations étaient dorénavant perçues non pas comme des endroits de ressourcement pour des victimes d'abus passives, mais comme des prisons pour des femmes violentes et dangereuses, de qui la société - familles, enfants, personnes âgées — devait être protégée. Le SCC, qui devait également composer avec les demandes du personnel et des syndicats, a réagi en installant davantage de clôtures, d'ailleurs plus hautes, ainsi que des caméras de surveillance; il a également doublé les espaces sécuritaires disponibles dans les nouvelles prisons et a mis le programme mère-enfant en attente. Sur la base des recommandations du rapport interne sur l'incident d'avril 1994, et malgré la fermeture prévue de la prison des femmes en 1996, des cellules d'isolement supplémentaires ont été construites dans la prison.

\section{Vers une redéfinition des choix et de la faisabilité}

En septembre 1996, une décision critique a été prise, celle d'exclure toutes les femmes classées à sécurité maximale des nouvelles prisons. Celles-ci seraient plutôt installées dans les institutions à sécurité maximale pour hommes, dont le pénitencier de Kingston, d'où elles avaient été retirées au début du siècle. Au sein du SCC, l'idée de séparer les «femmes 
difficiles» des autres s'est imposée au fil des discussions. Dans cette catégorie de "femmes difficiles», on inclut celles qui étaient désignées comme "violentes et dérangeantes» ou «difficiles à gérer», celles à qui on attribuait une personnalité «borderline», ou qui souffraient de perturbations de l'identité ou de troubles de la personnalité (SCC, 1996).

Les revendications des détenues, des bénévoles et des avocats à propos des injustices liées à l'événement d'avril 1994 ont été ignorées ou écartées par le SCC. Un rapport de l'Enquêteur correctionnel (1995) et la diffusion télévisée de l'équipe d'intervention effectuant des fouilles sur les femmes ont amené le Solliciteur général à mettre immédiatement sur pied une enquête indépendante menée par la juge Louise Arbour. Son rapport de 1996 a fait ressortir une indifférence totale, de la part des acteurs impliqués, à l'endroit des droits des détenues et une culture de la défensive dans le système correctionnel. Il est ironique que ces événements ait eu lieu au moment où les nouveaux régimes centrés sur les femmes étaient développés de façon active. Comme le concluait Louise Arbour à partir des propos tenus par le SCC à la Commission, «these comments brought home to me the realization that, despite its recent initiative, the Correctional Service resorts invariably to the view that women's prisons are, or should be, just like any other prison» (Arbour, 1996 : 178).

La juge Arbour appuyait la philosophie globale de La création de choix, tout particulièrement l'idée du pavillon de ressourcement. Elle a émis quatorze recommandations visant, entre autres, à construire des protections légales et une «culture des droits» au sein du SCC. À ce jour, seules quelques recommandations de son rapport ont été appliquées (HannahMoffat et Shaw, 2000b).

La décision d'exclure toutes les femmes à sécurité maximale des nouvelles prisons ne sera pas révoquée avant 2002. Ces femmes, dont la moitié sont autochtones, ont été placées en isolement dans des prisons pour hommes à travers le pays. Le pavillon de ressourcement, prévu en tant qu'endroit de guérison dont toutes les femmes autochtones pourraient se prévaloir, n'est toujours pas une option pour les femmes classées à sécurité maximale. Ces femmes, tout comme celles qui éprouvent des problèmes de santé mentale, ont été placées dans des institutions pour hommes. Sans même compter l'absence de programmes et de services appropriés, elles ont vécu des conditions que l'on peut croire pires que celles qui prévalaient à la prison des femmes avant la commission spéciale (Morin, 1999). 


\section{Une vision limitée de la réalité des contrevenantes}

Le Groupe d'étude n'a pas porté suffisamment attention à la question de la violence des femmes, ayant plutôt choisi de présenter ces dernières comme des victimes de violence avant tout. Cette décision a eu des répercussions importantes. Elle a au moins eu pour effet de laisser le SCC dans l'ombre, sans ressources pour comprendre les besoins des femmes qui ont effectivement recours à la violence - et y répondre. Les événements d'avril 1994 ont fait ressortir le profil d'un petit groupe que l'on a fini par désigner comme étant un groupe «difficile à gérer». Les comportements passés et anticipés de ces femmes ont été utilisés pour justifier un certain nombre d'interventions punitives traditionnelles, et pour légitimer, dans le discours public, le recours à ces méthodes. Une nouvelle stratégie nationale ${ }^{5}$ pour les femmes à hauts risques - les femmes qui ont des besoins importants - est présentement mise en œuvre. La stratégie développée par le SCC inclut la poursuite de l'expansion des unités plus sécuritaires dans les prisons régionales, afin qu'il soit possible de recevoir jusqu'à 30 détenues à sécurité maximale. Des milieux de vie structurés ou «maisons de ressourcement » avec des employés spécialisés devaient être fonctionnels en septembre 2001 au sein de chaque prison régionale, pour les femmes qui nécessitaient un encadrement plus intensif que les autres détenues (Enquêteur correctionnel, 2000). Deux unités de thérapie dialectique du comportement, pour des traitements intensifs en santé mentale, sont aussi sur le point d'être construites. La Stratégie d'intervention intensive annoncée en septembre 1999 a fait l'objet de projets-pilotes au Saskatchewan Regional Psychiatric Centre (Mirasty et al., 2001), au Burnaby Correctional Centre for Women en ColombieBritannique et à la prison des femmes de Kingston.

Des modèles théoriques très différents et non féministes semblent s'être substitués à celui du Groupe d'étude pour expliquer le comportement des femmes ou évaluer leurs besoins. Les principes fondamentaux de La création de choix, soit ceux d'autonomisation et de choix significatifs, sont actuellement très peu appliqués à la situation des détenues. Celles qui participent aux régimes de santé mentale ou à la stratégie de guérison intensive doivent y prendre part; on ne leur laisse pas le choix. Comme Hannah-Moffat l'a soutenu ailleurs (2001), la notion féministe d'auto-

5. Pour une élaboration détaillée de la logique fondatrice et des techniques utilisées pour les femmes «difficiles à gérer», voir Whitehall, 1995 ; Rivera, 1996; Laishes, 1997; Warner, 1998; McDonagh, 1999; Mirasty et al., 2001. 
nomisation a été réinterprétée dans le contexte carcéral comme une responsabilisation - des choix que les femmes doivent faire si elles veulent progresser à travers les niveaux de sécurité, obtenir des privilèges ou accéder à une libération conditionnelle.

Même si le pavillon de ressourcement a été la seule institution exclue de ces derniers développements, les femmes autochtones qui ont un classement sécuritaire maximum sont encore loin d'y avoir accès. Plusieurs des personnes impliquées dans son développement sont considérablement préoccupées par l'incompatibilité de la notion de guérison avec le système correctionnel et par l'expropriation de la spiritualité autochtone (Morin, 1999; Hayman, 2000; Monture, 2000). Même si, pour le SCC, la nouvelle stratégie se veut une "alternative plus humaine» pour les femmes à sécurité maximale et celles qui ont des besoins plus criants en santé mentale, cette stratégie risque d'avoir un impact considérable sur l'atmosphère et les pratiques quotidiennes des établissements régionaux. Les femmes à sécurité minimale ou moyenne vont entre autres devoir composer avec un espace plus restreint et un accès plus limité aux installations. Cette stratégie symbolise la continuité des stratégies de gestion pénale punitives et quasi thérapeutiques (Bertrand et al., 1998). Cela était peut-être prévisible (Hannah-Moffat, à paraitre) mais marque néanmoins une rupture avec la vision du Groupe d'étude. Rétrospectivement, $L a$ création de choix semble avoir eu une vision limitée de la réalité dans sa caractérisation des femmes détenues basée sur la victimisation. Elle n'a pas réussi à traiter et à prévoir ce qui semble maintenant une évidence, à savoir que, lorsque les femmes en prison résistent par la violence dirigée vers l'extérieur et par l'automutilation, la prison réagit. En l'absence d'une alternative clairement définie, les réponses apportées à la violence des femmes sont inspirées des anciennes techniques, celles que la commission a déjà rejetées comme étant inappropriées.

Le SCC a développé de nouvelles techniques et de nouveaux raisonnements pour la gestion des «détenues résistantes» qui pourraient présenter un risque pour la sécurité du public et/ou pour le «bon ordre des institutions». Ces nouveaux développements ne sont cependant pas perçus comme étant en contradiction avec la philosophie plus large de l'autonomisation. Le groupe de détenues «difficile», donc récalcitrant, n'est pas présenté comme étant sensible aux principes énoncés par la commission. La résurgence d'images de femmes «folles» et «mauvaises» a permis l'intensification de stratégies punitives et une apathie correspondante envers le contrevenant à sécurité minimale. Onze ans après La création de choix, on 
se retrouve avec cinq nouvelles prisons, des niveaux de sécurité beaucoup plus élevés puis une nouvelle stratégie, de nouveaux régimes et de nouvelles installations pour gérer «les difficiles ou perturbées». À propos de la stratégie communautaire, cependant, il n'y a pas eu beaucoup plus que des discussions. Aucune installation comparable à celles qui sont disponibles pour les hommes à sécurité minimale n'a été fournie.

\section{Qu'est-il advenu de la sécurité minimale et de la stratégie communautaire?}

L'absence de suites aux recommandations concernant la stratégie communautaire est l'un des manques les plus évidents et surprenants de la mise en œuvre de La création de choix. Le Groupe d'étude avait mis l'accent sur le fait que les femmes avaient beaucoup moins accès que les hommes aux ressources communautaires pour faciliter leur réintégration. Il a revendiqué plus de centres communautaires de libération, de maisons de transition, d'appartements satellites et de centres administrés par des groupes ou communautés autochtones (La création de choix, 1990 : 126-128). Il a également proposé des options créatives pour les femmes provenant de petites communautés isolées, celles qui étaient incapables de vivre en groupe et celles qui avaient des besoins spéciaux. Aucun fond gouvernemental ne fut octroyé pendant la mise en œuvre du rapport pour développer des services communautaires - soit la deuxième partie du plan, le complément essentiel des nouvelles institutions régionales.

Un court document de travail a été produit en 1998, certaines consultations ont été tenues avec les groupes d'intérêts de la fin des années 1990, et du financement a été attribué à des projets individuels afin de soutenir de «nouvelles» initiatives, telles que les placements dans des maisons privées. Les sections 81 et 84 de la Loi sur le système correctionnel et la mise en liberté sous condition concernant les alternatives communautaires pour les contrevenants autochtones n'ont pas mené à un nombre significatif d'initiatives pour les femmes. Face aux problèmes de l'offre insuffisante de services appropriés puis de l'absence de réseaux de soutien et de programmes pour les femmes qui sortent de prison, il n'y a aucune solution en concertation qui soit en vue. Les alternatives non carcérales pour les femmes continuent à être éparpillées, sous-financées et insuffisantes (il y a beaucoup plus d'intéressées que de places disponibles). Alors qu'une nouvelle maison de transition pour femmes a ouvert 
ses portes à Calgary, celle d'Ottawa est fermée depuis 1999 à cause de coupures dans le financement (provincial) (CAEFS, 1999).

Presque $50 \%$ de la population des prisons régionales est classée à un niveau de sécurité minimal (SCC, 2000). Les femmes risquent davantage d'être classées à leur admission — pour le deux tiers d'entre elles, il s'agit de la première classification de sécurité. Ces femmes sont placées dans un milieu beaucoup plus sécuritaire que ne la justifie leur classification. Cela est évident lorsqu'on les compare avec les détenus fédéraux de genre masculin du même niveau de sécurité. Dans une proportion importante, ces derniers vivent dans des établissements sans clôture extérieure, ils ont des responsabilités individuelles plus importantes que les femmes et ils peuvent bénéficier d'options de travail à leur libération. Le seul établissement à sécurité minimale spécifiquement créé pour (dix) des détenues de niveau fédéral, la Isabel McNeill House, doit fermer en 2002. Cela signifie que toutes les détenues à sécurité minimale vont demeurer dans des établissements régionaux à plusieurs niveaux de sécurité, lesquels sont en réalité des institutions à sécurité moyenne.

La situation des femmes est caractérisée par le manque d'installations communautaires. Le niveau de sécurité minimal est un statut qui doit être mérité par celles qui ne sont pas classées comme tel initialement. On peut soutenir que les femmes n'ont pas vraiment intérêt à s'efforcer de mériter ce statut alors qu'il n'y a qu'une faible différence entre les conditions des niveaux de sécurité minimal et moyen. Elles ont également moins accès aux programmes et services dans la communauté. Le succès d'une libération communautaire dépend pourtant de trois aspects essentiels et interdépendants : la planification et l'application des absences temporaires; les libérations de jour et la pleine libération conditionnelle; enfin, l'accès à des conditions de libération adéquates. Il est essentiel que les femmes puissent, à leur retour en communauté, disposer d'un endroit pour vivre, avoir accès à des programmes appropriés, à un emploi ou à une formation. La présence de réseaux de soutien tels que la famille, les amis et les employés des libérations conditionnelles, ou les employés correctionnels du secteur communautaire, est également primordiale. La disponibilité de ces derniers devrait d'ailleurs aller bien au delà de la date d'expiration du mandat.

\section{L'avenir du traitement pénal au féminin}

Globalement, les nouvelles prisons régionales ont connu une augmentation substantielle de leur capacité. Le nombre de lits s'est accrû en raison 
des problèmes de surpopulation, lesquels résultent en partie du manque d'établissements à sécurité minimale et du manque de ressources pour les femmes libérées dans la communauté, et en partie de transformations dans les modèles de «sentencing». Dans certaines provinces, les tribunaux semblent avoir davantage recours aux sentences fédérales pour les femmes afin qu'elles puissent avoir accès à des programmes qui ne sont pas disponibles dans les prisons provinciales. Ainsi, la capacité des prisons s'est accrue à un moment où le nombre de femmes qui reçoivent une sentence fédérale décroît. Ce phénomène est complexe, mais il met tout de même en évidence l'échec de la stratégie communautaire. Aucune alternative significative à l'incarcération des femmes n'a été créée.

Pendant un certain temps, quelques femmes sous juridiction fédérale ont été placées dans des pénitenciers pour hommes à travers le pays, connaissant des conditions qui étaient équivalentes à celles qui prévalaient à la prison des femmes de Kingston, ou même pires. La prison des femmes a finalement fermé ses portes en juillet 2000. Cette année, les nouvelles installations destinées aux femmes à sécurité maximale ou ayant des problèmes de santé mentale seront mises en opération au sein des prisons régionales. Les prisons sont par ailleurs devenues plus bureaucratiques dans leurs pratiques quotidiennes, ce qui inclut l'adoption de protocoles d'évaluation construits pour les hommes (Hannah-Moffat et Shaw, 2000b). On a assisté à un retrait de certaines initiatives centrées sur les femmes et sensibles aux différences culturelles, initiatives dont on avait tenu compte au cours des premières années de la mise en œuvre du rapport. Les femmes perçues comme dérangeantes, agitées et difficiles à gérer sont mises à part et sujettes à des régimes qui different substantiellement de la vision de $\mathrm{La}$ création de choix. Les femmes à sécurité minimale continuent à vivre dans des conditions de sécurité plus élevées que la plupart des hommes de même niveau. Il n'y a eu aucune expansion des programmes et installations communautaires. Les femmes sous juridiction fédérale en ColombieBritannique ont en outre été exclues de plusieurs changements et demeurent présentement sous responsabilité provinciale ${ }^{6}$.

Il est clair que les idéaux de La création de choix étaient limités, en théorie et en pratique. La vision originale des réformateurs a néanmoins été transformée, et continue à l'être. On la modifie pour bien l'ajuster aux

6. Le gouvernement de Colombie-Britannique a annoncé la fermeture de la prison de Burnaby en janvier 2002; des discussions sont présentement en cours avec le SCC pour décider de l'avenir de cet établissement. 
agendas gouvernementaux et correctionnels. La notion autochtone de guérison et des notions féministes telles que l'autonomisation ont été absorbées par le système correctionnel et elles ont, en conséquence, perdu leur sens initial. Les conditions matérielles dans les nouveaux établissements au sein desquels vivent les détenues fédérales sont sans aucun doute bien meilleures qu'avant, mais des problèmes subsistent. Cet article visait à examiner différents aspects du monde de l'après Création de choix. Les événements des onze dernières années nous placent devant plusieurs dilemmes importants et non résolus. Les membres du Groupe d'étude ont fait un travail innovateur, mais ils ont surestimé le potentiel de changement du système correctionnel. Les participants du Groupe étaient cependant loin d'être naïfs. Ils étaient certainement conscients des limites avec lesquelles ils devaient composer, mais leur vision du changement a dépassé la capacité du système. Les réalités bureaucratiques de la peine et la tendance punitive, orientée vers des solutions carcérales, qui caractérisent depuis longtemps la société a restreint la croissance de cette vision.

Même si la stratégie a quelques avantages, la logique correctionnelle centrée sur les femmes qui est actuellement appliquée ne peut pas fondamentalement changer l'emprisonnement. De telles réformes pénales ne remettent pas en question le caractère central de l'incarcération. Ironiquement, le régime des prisons, qui avait déjà échoué à fournir une étendue appréciable de programmes et de formes de soutien pour les femmes, offre maintenant encore plus de modèles individualisés et psychiatriques, mais cette fois sous le couvert de l'autonomisation. L'éventuel échec du nouveau régime centré sur les femmes et sensible aux différences culturelles, régime qui devait permettre aux femmes d'avoir plus de contrôle sur leur vie et leur offrir une opportunité de guérison, ne devrait pas être une surprise. Les prisons peuvent permettre des changements et être par moments flexibles, mais elles peuvent aussi résister à, incorporer, redéfinir et absorber les discours critiques. Finalement, nous pouvons seulement espérer que la mise en œuvre de La création de choix représente les premiers pas vers une alternative imparfaite.

\section{Références}

Arbour, L. (1996). Commission of inquiry into certain events at the prison for women in Kingston. (Arbour Report). Ottawa : Public Works and Government Services of Canada.

Bertrand, M.-A., Biron, L., di Pisa, C., Fagnan, A., \& McLean, J. (1998). Prisons pour femmes. Montréal : Éditions du Méridien. 
Berzins, L., \& Collette-Carrière, R. (1979). La femme en prison : un inconvénient social! Santé mentale au Québec, 4 (2), 87-103.

Berzins, L., \& Hayes, B. (1987). The diaries of two change agents. In E. Adelberg \& C. Currie (eds), Too few to count: Canadian women in conflict with the law. Vancouver : Press Gang Publishers.

Biron, L. (1992). Les femmes et l'incarcération. Le temps n'arrange rien. Criminologie, $X X V(1), 119-134$.

Canadian Association of Elizabeth Fry Societies - CAEFS (1999). Annual report. Ottawa : Canadian Association of Elizabeth Fry Societies.

Cooper, S. (1987, 1993). The evolution of the federal women's prison. In E. Adelberg \& C. Currie (eds), Too few to count: Canadian women in conflict with the law. Vancouver : Press Gang Publishers.

Correctional Investigator (2001). Annual report of the correctional investigator 19992000. Ottawa : Ministry of Public Works Canada.

Correctional Service of Canada (1994). Draft correctional program strategy for federally sentenced women - Abridged version. Ottawa : Correctional Services of Canada.

Correctional Service of Canada (1995). Briefing notes-Strategy to manage federally sentenced women who behave violently. Ottawa: The Federally Sentenced Women Program.

Correctional Service of Canada (1996). The correctional strategy. Ottawa : Correctional Service of Canada-Federally Sentenced Women Program.

Correctional Service of Canada (2000). Statistical overview-Women offenders. Ottawa : Correctional Service of Canada -Women Offender Section (April).

Drouin, M.-A. (1997). Rethinking women's imprisonment: A Canadian perspective. In S. Hayman (ed.), Imprisoning women: Recognizing differences. Proceedings of a Conference. London : Institute for the Study and Treatment of Delinquency.

Faith, K. (1993). Unruly women: The politics of confinement and resistance. Vancouver : Press Gang Publishers.

Faith, K. (1995). Aboriginal women's Healing Lodge: Challenge to penal correctionalism? The Journal of Human Justice, 6 (2), 79-104.

Faith, K. (1999). Transformative justice versus re-entrenched correctionalism: The Canadian experience. In S. Cook \& S. Davies (eds), Harsh punishment: International experiences of women's imprisonment. Boston : Northeastern University Press.

Frigon, S. (1999). Une radioscopie des événements survenus à la Prison des femmes de Kingston en Ontario en avril 1994 : la construction d'un corps dangereux et d'un corps en danger. Canadian Women's Studies/ Les cabiers de la femme, $19(1-2)$.

Hannah-Moffat, K. (1995). Feminine fortresses: Woman-centred prisons? The Prison Journal, 75 (2), 135-164.

Hannah-Moffat, K. (2000a). Prisons that empower: Neoliberal governance in Canadian women's prisons. British Journal of Criminology, 40, 510-551.

Hannah-Moffat, K. (2000b). Re-forming the prison-rethinking our ideals. In K. Hannah-Moffat \& M. Shaw (eds), An ideal prison? Critical essays on women's imprisonment in Canada. Halifax : Fernwood. 
Hannah-Moffat, K. (2001). Punishment in disguise: Governance in canadian women's prisons. Toronto : University of Toronto Press.

Hannah-Moffat, K. (2002). Lessons learned: Some reflections on federal women's prison re-form. In P. Carlen (ed.), Women and Punishment. UK : Willan Publishing.

Hannah-Moffat, K., \& Shaw, M. (2000a). An ideal prison? Critical essays on women's imprisonment in Canada. Halifax : Fernwood.

Hannah-Moffat, K., \& Shaw, M. (2000b). Gender, diversity, risk assessment and classification with federally sentenced women. Ottawa : Status of Women Canada.

Hannah-Moffat, K., \& Shaw, M. (2001). Situation risquée : le risque et les services correctionnels au Canada. Criminologie, 34 (1), 47-72.

Hamelin, M. (1989). Femmes et prison. Montréal : Éditions du Méridien.

Hayman, S. (2000). Prison reform and incorporation: Lessons from Britain and Canada. In K. Hannah-Moffat \& M. Shaw (eds), An ideal prison? Critical essays on women's imprisonment in Canada. Halifax : Fernwood Publishing.

Hayman, S. (à paraître) Imprisoning our sisters: The incorporation of conscience. University of London.

Kendall, K. (2000). Psy-ence fiction: The moral regulation of female prisoners through psychological sciences. In K. Hannah-Moffat \& M. Shaw (eds), An ideal prison? Critical essays on women's imprisonment in Canada. Halifax : Fernwood Publishing.

Laishes, J. (1997). Mental health strategy for women offenders. Ottawa : Federally Sentenced Women's Program, Correctional Service of Canada.

Laishes, J., \& Lyth, S. (1996). Intensive healing (mental bealth) program. Ottawa : Correctional Service of Canada-Federally Sentenced Women Program.

Mandarake-Sheppard, A. (1986). The dynamics of aggression in women's prisons in England and Wales. London : Gower.

McDonagh, D. (1999). Federally sentenced women - Maximum security interview project- Not letting the time do you. Ottawa : Federally Sentenced Women's Program.

Mirasty, M., Presse, C., Roth, B., \& Thompson, H. (2001). Para suicidal behaviours bandbook for forensic inpatients - Intensive healing program for women Regional Psychiatric Centre. Saskatoon : Regional Psychiatric Centre.

Monture-Angus, P. (2001). Aboriginal women and correctional practice: Reflections on the Task Force on Federally Sentenced Women. In K. Hannah-Moffat \& M. Shaw (eds), An ideal prison? Critical essays on women's imprisonment in Canada. Halifax : Fernwood Publishing.

Morin, S. B. (1999). Federally sentenced aboriginal women in maximum security What happened to Creating Choices? Ottawa : Correctional Service of Canada.

Pollock-Byrne, J. (1990). Women, prison and crime. Pacific Grove, CA : Brooks/Cole.

Reynolds, B. (1996). Federally sentenced women in Canada. Ottawa : Correctional Service of Canada. Communication présentée à la Commonwealth Corrections Administrator's Conference, 25-28 novembre, Australie.

Rivera, M. (1996). Giving us a chance: Needs assessment mental bealth resources for federally sentenced women in the regional facilities. Ottawa : Correctional Service of Canada. 
Shaw, M. (1991). The female offender: Report on a preliminary study. Ottawa : Ministry of the Solicitor General.

Shaw, M. (1993). Reforming federal women's imprisonment. In E. Adelberg \& C. Currie (eds), In conflict with the law. Vancouver : Press Gang Publishers.

Shaw, M. (1994a). Ontario women in conflict with the law. Toronto : Ministry of Solicitor General and Correctional Services.

Shaw, M. (1994b). Women in prison: A literature review. Forum, 6 (1), Special issue on women in prison. Ottawa : Correctional Service Canada.

Shaw, M. (1995). Conceptualizing violence by women. In R. Dobash, R. Dobash \& L. Noakes (eds), Gender and crime. Cardiff : University of Wales Press.

Shaw, M. (1996). Is there a feminist future for women's prisons. In R. Mathews \& P. Francis (eds), Prisons 2000: An international perspective on the current state and future of imprisonment. Basingstoke : Macmillan Press.

Shaw, M. (1999). A video camera can change your life. In S. Cook \& S. Davies (eds), Harsh punishment: International experiences of women's imprisonment. Boston : Northeastern University Press.

Shaw, M. (2000). Women, violence and disorder in prisons. In K. HannahMoffat \& M. Shaw (eds), An ideal prison? Critical essays on women's imprisonment in Canada. Halifax : Fernwood Publishing.

Sim, J. (1990). Medical power in prisons. Milton Keynes : Open University Press.

Stableforth, N. (1997). Women offenders. In Let's Talk. Ottawa : Correctional Service of Canada.

Statistics Canada (1998). A one-day snapshot of inmates in Canada's adult correctional facilities. Juristat, 18 (8). Ottawa : Canadian Centre for Justice Statistics.

Statistics Canada (1999). Female inmates, aboriginal inmates, and inmates serving life sentences: A one-day snapshot. Juristat, 19 (5) Ottawa : Canadian Centre for Justice Statistics.

Sugar \& Fox (1989). Nistum peyako séht'wawin iskwewak: Breaking the chains. Canadian Journal of Women and the Law, 3, 465-482.

Task Force on Federally Sentenced Women (1990). Report of the Task Force on Federally Sentenced Women - Creating Choices. Ottawa : Ministry of the Solicitor General.

Warner, A. (1998). Implementing choices at regional facilities: Program proposals for woman offenders with special needs. Ottawa : Correctional Services of Canada.

Whitehall, G. C. (1995). Mental health profile and intervention strategy for Atlantic region federally sentenced women. Ottawa : Correctional Service of Canada. 